\title{
The presence of genetic hypertension stimulates early renal accumulation of fibronectin in experimental diabetes mellitus
}

\author{
A. E. Righetti, P. A. Boer-Lima, J. B. Lopes de Faria \\ Division of Nephrology, Faculty of Medical Sciences, State University of Campinas, São Paulo, Brazil
}

\section{Abstract}

Aims/hypothesis. To investigate the interaction between hypertension and diabetic nephropathy, we studied the renal accumulation of fibronectin in a genetic model of hypertension with streptozotocin-induced diabetes mellitus.

Methods. Spontaneously hypertensive rats (SHR) and their genetically normotensive control Wistar Kyoto rats (WKY) were studied at 4 weeks of age. The rats were killed 20 days after the induction of diabetes mellitus. The renal accumulation of fibronectin was estimated using Western blot analysis as well as immunofluorescence technique and confocal microscopy.

Results. Blood glucose concentrations were similar in diabetic SHR rats $(27 \pm 3.3 \mathrm{mmol} / \mathrm{l})$ and WKY rats $(25.5 \pm 2.7 \mathrm{mmol} / \mathrm{l})$. The systolic blood pressure was higher in both groups of SHR rats than in the control rats. The abundance of renal fibronectin as detected by Western blot analysis was $(p<0.05)$ higher in the diabetic SHR rats $(41.4 \pm 15.0$ densitometric U, $n=8)$ than in the control rats, and no difference was observed between diabetic and control WKY rats $(20.8 \pm 6.2, n=8)$ and $(27.8 \pm 17.2, n=8)$. The mean peak intensity of fibronectin signal within the glomerulus as estimated by confocal microscopy was higher $(p<0.05)$ in the diabetic SHR rats $(32.9 \pm 3.5)$ than in control SHR rats $(11.9 \pm 5.7)$ or diabetic $(7.4 \pm 2.2)$ and control $(15.2 \pm 7.9) \mathrm{WKY}$ rats.

Conclusion/interpretation. In experimental diabetes the presence of genetic hypertension promotes earlier accumulation of renal fibronectin, a matrix protein implicated in renal glomerulosclerosis. [Diabetologia (2001) 44: 2088-2091]

Keywords Hypertension, diabetes mellitus, fibronectin, diabetic glomerulosclerosis, diabetic nephropathy, predisposition to hypertension, confocal microscopy.
Both in humans and animal models of diabetes mellitus a long period of hyperglycaemia is required for the development of the characteristic morphological lesion of diabetic nephropathy $[1,2]$. However, the morphological lesions are likely to be end points of biological process that have become operative well in advance of the appearance of architectural modifi-

Received: 11 January 2001 and in revised form: 30 May 2001

Corresponding author: Dr. José B. Lopes de Faria, Division of Nephrology, Faculty of Medical Sciences, State University of Campinas, SP, Brazil, e-mail: jblfaria@fcm.unicamp.br Abbreviations: SHR, Spontaneously hypertensive rat; WKY, Wistar Kyoto; K kiloDaltons; AER, albumin excretion rate cations in the glomeruli. It is also possible that the time-course of these processes could be modified by factors other than hyperglycaemia. Measuring early kidney biosynthetic changes relevant to the lesions of diabetic nephropathy in animal models can assess these possibilities.

In patients with Type I (insulin-dependent) diabetes mellitus hypertension and microalbuminuria are the earliest and concomitant clinical manifestations of diabetic renal disease [3]. It has even been suggested that hypertension contributes to the pathogenesis of diabetic renal disease [4]. In experimental diabetes the presence of hypertension has been shown to anticipate the increase in the albumin excretion rate [2]. However, in this last study a period of 32 weeks 
of severe hypertension and diabetes was necessary before abnormalities in the glomerular structure could be demonstrated [2]. We thus investigated whether the presence of a short period of mild genetic hypertension and diabetes would modify the amount of renal fibronectin, an extracellular matrix component that increases in the early phase of diabetic renal disease [5]. We chose the spontaneously hypertensive rats (SHR), a widely studied model of essential hypertension, which has a normotensive period (the first 4 weeks of life) before the genetically determined development of hypertension, which occurs in $100 \%$ of the animals with 12 weeks of age ([6].

\section{Material and methods}

The protocol for this study complies with the guidelines for the care and use of laboratory animals, published by the National Institutes of Health. All reagents were purchased from Sigma, St Louis, Mo., USA, unless stated otherwise. We derived the SHR and their genetically normotensive control Wistar Kyoto (WKY) rats from animals supplied by Taconic (Germantown, N. Y., USA) and bred them in our animal facility. To avoid the presence of pre-existing hypertension in the SHR that could in itself influence the expression of renal fibronectin [7], diabetes was induced in male SHR and WKY rats who were 4 weeks of age by an injection with $65 \mathrm{mg} / \mathrm{Kg}$ of streptozotocin in the tail vein. Control groups received only vehicle. Blood glucose concentrations were measured and only values above $15 \mathrm{mmol} / \mathrm{l}$ were considered for the experiments. Systolic blood pressure was measured by tail-cuff plethysmography. One day before being killed, urine was collected from the rats who had been placed in individual metabolic cages for $24 \mathrm{~h}$ to measure the albumin excretion rate (AER) by single radial immunodiffusion [8]. The interassay coefficient of variation was $8.2 \%$ at a concentration of $20.7 \mu \mathrm{g} / \mathrm{ml}$ and the detection limit of the assay was $3 \mu \mathrm{g} / \mathrm{ml}$. Rats were killed 20 days after the injection of streptozotocin or citrate buffer and the renal expression of fibronectin was determined by Western blot analysis. Twenty micrograms of total protein from renal cortices were analysed by $(6 \%)$ SDS-PAGE followed by nitrocellulose transfer using Mini-Protean II Dual Slab Cell apparatus (Bio-Rad Laboratories, Hercules, Calif., USA). The membranes were incubated with the primary, a goat anti-rat fibronectin antibody (Calbiochem-Novabiochem, La Jolla, Calif., USA), and with the antigoat secondary antibody conjugated to horseradish peroxidase. Immunoreactive bands were made visible using the enhanced chemiluminescence method (SuperSignalCL-HPR Substrate System; Pierce, Rockford, Ill., USA). Exposed films were scanned with a laser densitometer (Bio-Rad) and quantitatively analysed with Multi-Analyst Macintosh Software for Image Analysis Systems (Bio-Rad).

To investigate if there were differences in glomeruli accumulation of fibronectin among experimental groups, immunofluorescence studies were done. To avoid confounding influences by plasma fibronectin trapped in the tissue, the right kidney was perfused in vivo initially with saline followed by a fixative solution of Methacarn [9] and embedded in paraffin. Fivemicrometer sections were de-waxed, washed in PBS ( $\mathrm{pH} 7.4$ ) and placed in $5 \%$ non-fat milk in PBS. Tissue sections were incubated overnight at $4{ }^{\circ} \mathrm{C}$ with a rabbit anti-rat fibronectin antibody (Calbiochem) and with FITC-conjugated anti-rabbit antibody. Immunoreactivity was assessed using a Zeiss fluores-
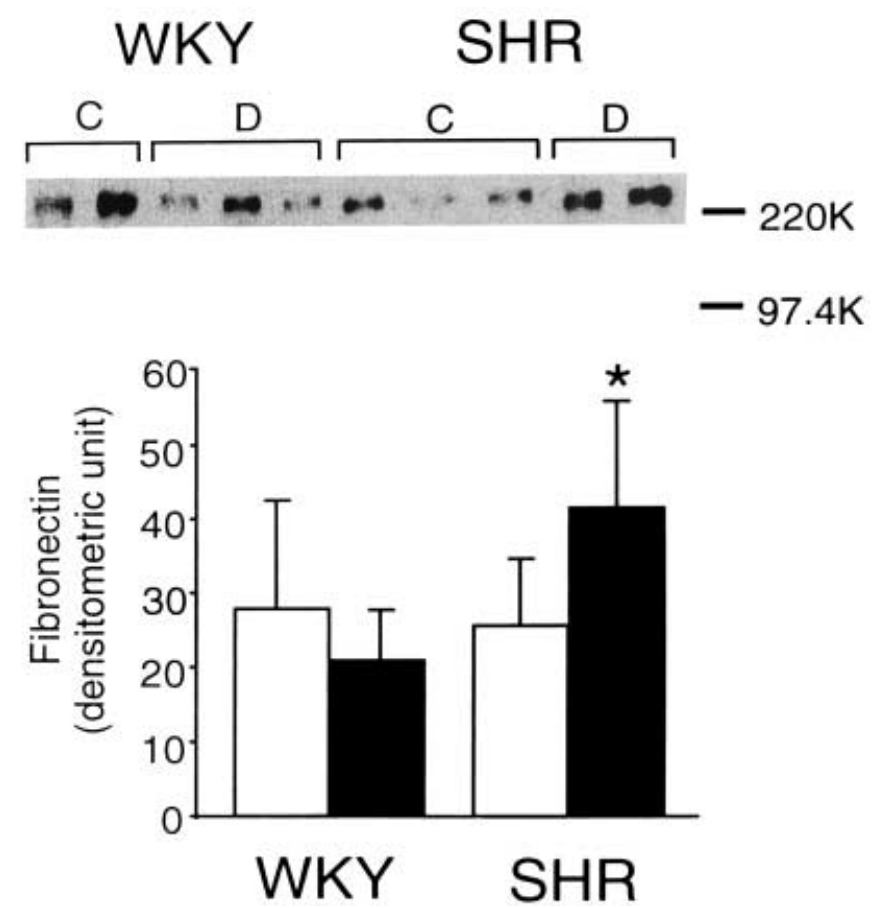

Fig. 1. Fibronectin protein in kidney of WKY and SHR. A Immunological detection (Western blot) of kidney cortex fibronectin. An experiment is shown where $20 \mu \mathrm{g}$ of lysates of kidney cortex from individual rats (c, control and D, diabetic rats) were subjected to PAGE and immunoblotted with antirat fibronectin antibody as described. Designated molecular weight markers are myosin $(220 \mathrm{~K})$ and phosphorylase b (97.4 $\mathrm{K})$. B Summary of densitometric analyses of fibronectin in kidney of control $(\square)$ and diabetic ( $\square$ ) WKY and SHR, $* p=0.05$ compared to all other groups (Fisher least significant difference method). The whole-model level of significance for ANOVA was $p=0.0228$. Data are given as means \pm SEM

cence microscope (Carl Zeiss, Jena, Germany) and a Bio-Rad MRC 1024 laser scanning confocal microscopy (Bio-Rad). The images of 10 consecutive cortical glomeruli of each rat obtained at single optical sections (same laser settings, same depth of sectioning) were stored and analysed using the accompanying software. To compare fluorescence intensities between the different experimental groups the area of the glomerulus was delineated and the mean pixel intensity (on a scale of 0 to 255) was obtained. The mean pixel intensity of 10 glomeruli was determined for each rat and used for statistical analysis.

Statistical analysis. AER was analysed after logarithmic transformation. Data are summarized as means \pm SEM except for AER, which are shown as geometric means and ranges. Oneway analysis of variance (ANOVA) was done for each variable to examine potential differences in the studied groups. Comparisons of group means were carried out by Fisher's least significant difference method using the StatView programme on a Macintosh computer (Cupertino, Calif., USA). Statistical significance was defined as a $p$ value of less than 0.05 .

\section{Results}

Weight gain was $(p<0.05)$ reduced in both strains of diabetic rats compared with non-diabetic rats $(101 \pm$ 

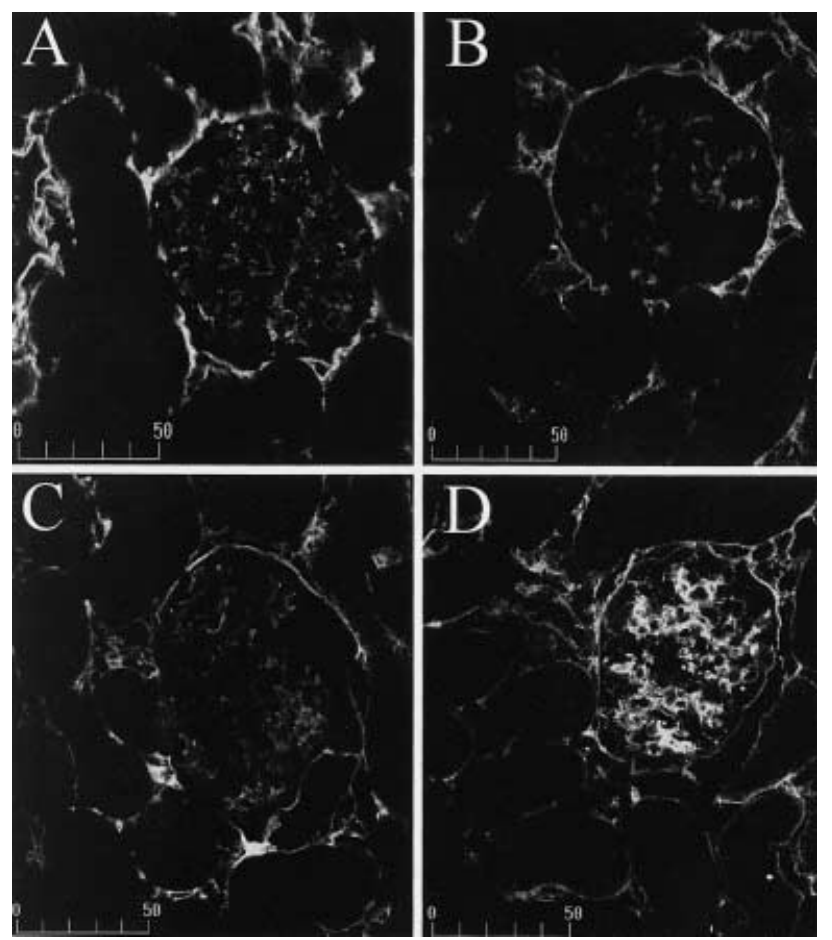

Fig. 2. Confocal fluorescent micrographs of anti-fibronectin immunoreactivity in glomeruli of control $(\mathbf{A})$ and diabetic $(\mathbf{B})$ WKY and control (C) and diabetic (D) SHR. Bar $=50 \mu \mathrm{m}$

23 vs $141 \pm 16 \mathrm{~g}$ and $123 \pm 25$ vs $180 \pm 23 \mathrm{~g}$, for SHR and WKY, respectively) 20 days after the injection of streptozotocin. Blood glucose was similar in diabetic SHR rats and WKY rats $(27 \pm 3.3$ vs $25.5 \pm 2.7 \mathrm{mmol} / \mathrm{l}$, respectively). The systolic blood pressure was higher in both groups of SHR rats than in the WKY rats $(148.8 \pm 7.9$ vs $121.8 \pm 7.9$ and $135.2 \pm 9.3$ vs $112.5 \pm 10.7 \mathrm{mmHg}$, for diabetic and control rats, and SHR and WKY, respectively). Diabetes was associated with $(p<0.05)$ increased AER over the 20 day study period in both rat strains (geometric mean [range], 224 [103-335] vs 442 [223-809] and 225 [135-451] vs 559 [237-720] $\mu \mathrm{g} / 24 \mathrm{~h}$, for control and diabetic rats, and SHR and WKY).

In renal cortex the level of immunoreactive fibronectin was $(p=0.05)$ higher in diabetic SHR rats $(41.4 \pm 15.0$ densitometric $\mathrm{U}, n=8)$ than in non-diabetic SHR rats $(25.6 \pm 9.4, n=7)$, although there was some variation between individuals rats (Fig. 1). The level of renal fibronectin was similar in diabetic WKY rats $(20.8 \pm 6.2, n=8)$ and control rats $(27.8 \pm 17.2, n=8)$. In perfused kidney, the accumulation of fibronectin within the glomerulus was markedly higher in the diabetic SHR rats than in the other groups (Fig. 2). A confocal quantitative study showed that the mean peak intensity of fibronectin signal in the glomeruli was higher $(p=0.05)$ in the diabetic SHR rats $(32.9 \pm 3.5, n=3)$ than in the control SHR rats $(11.9 \pm 5.7, n=3)$ or diabetic $(7.4 \pm 2.2, n=3)$ and control $(15.2 \pm 7.9, n=3) \mathrm{WKY}$ rats.

\section{Discussion}

This study shows that increased accumulation of fibronectin in the kidney is probably one of the initial events in the evolution of renal disease in a genetic model of hypertension (SHR) with streptozotocin-induced diabetes. These observations suggest that an early rise in glomeruli fibronectin could be one of the mechanisms by which the presence of predisposition to hypertension, or hypertension magnifies the risk of renal disease in individuals with diabetes. Of note, an increase in the magnitude and distribution of fibronectin is the hallmark of most human and experimental models of glomerulosclerosis, including diabetic glomerulosclerosis $[5,10]$.

The higher expression of fibronectin in diabetic SHR rats cannot be attributed to differences in metabolic control because the amount of glycaemia was similar in diabetic SHR rats and in WKY rats. However, in diabetic SHR rats, we were not able to separate clearly the influence of predisposition to hypertension from that of hypertension in itself, because our diabetic SHR rats were already hypertensive. Nevertheless, neither hypertension nor diabetes were able to magnify the renal fibronectin, because the expression of this matrix protein was not increased in control hypertensive SHR or diabetic WKY rats. In our study, the presence of predisposition to hypertension or increased blood pressure and diabetes were required for the observed increased in renal fibronectin. Prevention of the development of hypertension in diabetic SHR rats could allow us to identify the contribution of predisposition to hypertension as opposed to hypertension itself in the increased expression of fibronectin. In contrast to the accumulation of renal fibronectin, diabetes itself was able to induce increased permeability of albumin independently of the presence of predisposition of hypertension or increased amounts of blood pressure because this parameter was higher in the normotensive diabetic control rats, the WKY rats.

In summary we have shown that in experimental diabetes the presence of genetic hypertension promotes earlier accumulation of renal fibronectin, a matrix protein thought to be involved in the development of renal glomerulosclerosis [5, 10].

Acknowledgements. This work was supported by grant number 97/00469-4 from Fundação de Amparo à Pesquisa do Estado de São Paulo (FAPESP). Ms. A. E. Righetti was on receipt of a scholarship from CAPES. We are grateful to Mrs L. Aikawa da Silveira and T. Holtz Theizen for technical assistance. We also thank Dr. K. Franchini for helping with the perfusion of the kidney and R. Alves for editing the manuscript. 


\section{References}

1. Fioretto P, Steffes MW, Sutherland DER, Mauer M (1995) Sequential renal biopsies in insulin-dependent diabetic patients: structural factors associated with clinical progression. Kidney Int 48: 1929-1935

2. Cooper ME, Allen TJ, O'Brien RC et al. (1988) Effects of genetic hypertension on diabetic nephropathy in the rat functional and structural characteristics. J Hypertens 6: 1009-1016

3. Microalbuminuria Collaborative Study Group, United Kingdom (1993) Risk factors for development of microalbuminuria in insulin-dependent diabetic patients: a cohort study. Br Med J 306: 1235-1239

4. Viberti GC, Keen H, Wiseman MJ (1987) Raised arterial pressure in parents of proteinuric insulin-dependent diabetics. BMJ 295: 515-517

5. Ziyadeh FN, Hoffman BB, Han DC et al. (2000) Longterm prevention of renal insufficiency, excess matrix gene expression, and glomerular mesangial matrix expansion by treatment with monoclonal antibody in $\mathrm{db} / \mathrm{db}$ diabetic mice. PNAS 97: 8015-8020

6. Okamoto K, Tabei R, Fukushima M et al. (1966) Further observations of the development of a strain of spontaneously hypertensive rats. Jpn Circ J 30: 703-716

7. Hamaguchi A, Kim S, Ohta K et al. (1995) Transforming growth factor- $\beta_{1}$ expression and phenotypic modulation in kidney of hypertensive rats. Hypertension 26: 199-207

8. Mancini GAO, Carbonaro JF (1965) Immunochemical quantitation of antigens by single radial immunodiffusion. Immunochemistry 2: 235-254

9. Andrews PM, Coffey AK (1984) A technique to reduce fixation artifacts to kidney proximal tubules. Kidney Int 25: 964-968

10. Dixon AJ, Burns J, Dunnill MS, McGee JO (1980) Distribution of fibronectin in normal and diseased human kidneys. J Clin Pathol 33: 1021-1028 